\title{
Comparative Study Between Direct Load Control and Fuzzy Logic Control Based Demand Response
}

\author{
Chittesth Veni Chandran \\ Technological University Dublin \\ Malabika Basu \\ Technological University Dublin, mbasu@tudublin.ie \\ Keith Sunderland \\ Technological University Dublin, keith.sunderland@tudublin.ie
}

Follow this and additional works at: https://arrow.tudublin.ie/engscheleart

Part of the Electrical and Computer Engineering Commons

\section{Recommended Citation}

Chandran, C., Basu, M.\& Sunderland, K. (2016). Comparative study between direct load control and fuzzy logic control based demand response. UPEC16: Proceedings of the 51st. International Universities Power Engineering Conference. doi:10.1109/UPEC.2016.8114090

This Conference Paper is brought to you for free and open access by the School of Electrical and Electronic Engineering at ARROW@TU Dublin. It has been accepted for inclusion in Conference papers by an authorized administrator of ARROW@TU Dublin. For more information, please contact arrow.admin@tudublin.ie, aisling.coyne@tudublin.ie,gerard.connolly@tudublin.ie.

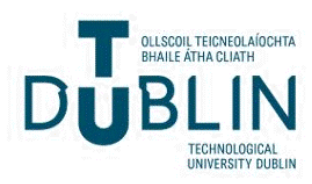




\section{Comparative Study between Direct Load Control}

\section{and Fuzzy Logic Control Based Demand Response}

\author{
Chittesh Veni Chandran \\ Dublin Institute of Technology, Ireland \\ School of Electrical Engineering Systems \\ chittesh.vc@mydit.ie
}

\author{
Malabika Basu \\ Dublin Institute of Technology, Ireland \\ School of Electrical Engineering Systems \\ malabika.basu@dit.ie
}

\author{
Keith Sunderland \\ Dublin Institute of Technology, Ireland \\ School of Electrical Engineering Systems \\ keith.sunderland@dit.ie
}

\begin{abstract}
Demand response (DR), as part of Demand side management (DSM), is a method that modifies consumer side energy consumption. By actively controlling the time associated with electricity consumption, both energy and economic efficiencies are increased. There is however, depending on the algorithm employed, potential for consumer comfort levels to be adversely affected. Generally direct load (Boolean logic) control is employed, which solely considers demand reduction and not customer comfort. In comparison, fuzzy logic based programs can consider non-deterministic inputs such as consumer comfort level with ease and simplicity. This paper considers the evolution of DR through a comparison of the classical (direct load method) and modern (fuzzy method) methods, with particular attention on the advantages provided by the latter. The impact on consumer comfort in fuzzy DR implementation is further explored through the effect of temperature deviation. In this regard, the consequences for fuzzy DR are considered in respect to three temperature profiles. Finally, temporal availability and the consequences for DR are considered in the context of a household.
\end{abstract}

Index Terms-Demand response, demand side management Direct Load control (DLC), Energy management, Energy savings, Fuzzy systems, Fuzzy control, Fuzzy technology, Load management, Power demand, Rational decision-making model,

\section{INTRODUCTION}

Electrical power systems, as complex active structures, require real time balancing of generation and demand to maintain stable and reliable operation. Furthermore, it is imperative that this balancing is implemented in a cost effective manner.

In earlier generation network configurations, production was abundant in meeting load demand and hence only basic load balancing algorithms were implemented and utilized. Essentially, demand balancing was facilitated by the supply side continuously adjusting generation. Nevertheless, by the 1970 's, the increasing demand for energy started to overtake the generation capacity of the system at peak demand. The solutions available were to either increase the generation capacity or to more efficiently manage load by using load management algorithms. Load management, termed demand side management (DSM), was the only economically feasible solution. This technique has subsequently been applied in various forms and levels [1]. It was first introduced by the Electric Power Research Institute (EPRI) in the 1980s as a series of activities that utilities can undertake to change their load shape and/or energy consumption pattern. These modifications were designed to provide the benefits of maximization of profit, investment delay, and reliability enhancement [1]. The decentralization of power systems and the liberalization of electricity markets have resulted in the evolution of DSM into two major groups [2]:
1. Energy efficiency, which deals with improving the energy utilization of services and products.

2. Demand response (DR), which is the process of changing the electricity usage patterns of end-use customers from their normal consumption patterns in response to high price, or an alternative incentive offered by the utility, to lower customer power usage when system reliability is jeopardized [2].

While technological hurdles were previously cited as one of the main challenges for DSM proliferation [4], this has changed due to technological advances in smart grids. The advancement of technologies such as grid-device bilateral communication, communication powered smart appliances, computationally powerful local controllers, cloud-based aggregation mechanisms and economically viable energy storage resources have solved the technological hurdles. Now it can be argued that finding a suitable business model is the next big challenge for DSM sustainability in a market-driven electricity system $[3,5]$.

Direct load control (DLC) is one of the most popular load control technologies, applied by the utilities to reduce the peak demand [6]. The method has evolved from simple frequency based load shedding to multi-layered aggregator responsebased load shedding. A load control technique, that effectively dips the voltage and frequency, is explained in [7] by Shuai Lu. However, this method does not account for the customer inconvenience or customer comfort. In addition, dynamic demand control [8] cannot be considered as an option for reducing peak demand. Naeem et al. assert that success of DR programs greatly depends on the consumer behaviour and comfort [9], but there are few examples in literature pertaining to customer behaviour/comfort and how DR is impacted in this regard. Examples of where customer comfort is prioritised in achieving energy efficiency include [10] and [11] where airconditioner control, as a representative element of customer comfort, is implemented through fuzzy logic. Other examples of non-fuzzy orientated control are found in [12] and [13]. In [12], a particle swarm optimization (PSO) based two level multi-agent controller is defined to optimize energy efficiency and customer comfort. In [13], an intelligent decision making model is developed using a dynamic notional price to increase energy efficiency that adapts to consumer preference in [13].

This paper evaluates the application of demand response into a domestic load control using two control techniques. DLC and fuzzy logic based DR are implemented and the change in DR with respect to the change in customer comfort is studied. Additionally, temporal availability of the household is modelled so that consideration to the change in DR with respect to change in temporal availability is provided. The paper will give a clear understanding of DR and its influencing 
parameters such that a fully functional and efficient demand response management controller can be developed.

The following sections provide discussion on the solution methodology as well as a full model description. The results pertaining to the analysis of field data where the modelling techniques are employed are also presented.

\section{Solution Methodology}

Demand response is implemented using two approaches, DLC and fuzzy logic. The DLC is considered as the classical representative method, whereas fuzzy approach represents the modern practice.

\section{A. Direct load control}

The DLC technique has been utilized for demand response implementation from the onset of load management program implementation. In this technique, the utility has remote access to certain individual consumer loads and DLC is remotely operated by the utility whenever needed. DLC schedules the loads in a house based on simple priority list. The method has been implemented in various forms and strategies. Generally, scheduling of load using a direct load method uses two approaches.

\section{Price based methods}

Price based methods utilize the day ahead price of electricity that is available through the electricity market or from the utility itself. According to the price per unit of electricity, a priority list is formulated for a set of consumer loads and the scheduling is organised accordingly. When the price is high, only high-priority devices are set to operate with the remaining (controlled) devices are 'off'. When the price is low all (listed) devices in the house are permitted to operate and hence no regulation or reduction is implemented. The general flow diagram is provided in fig 1 (a).

\section{Time of use based method}

This method utilizes the consumer basic load curve characteristics to identify the demand peaks and accordingly a schedule/priority list is made for modifying the consumption pattern. Usually the morning and evening times are designated as peak consumption times and during this time, fewer devices are operated. As illustrated in Fig. 1(b), the load regulation/reduction is conducted based on the time of peak occurrence.

While the above descriptions apply to the basic DR approach, numerous other methods and approaches are found

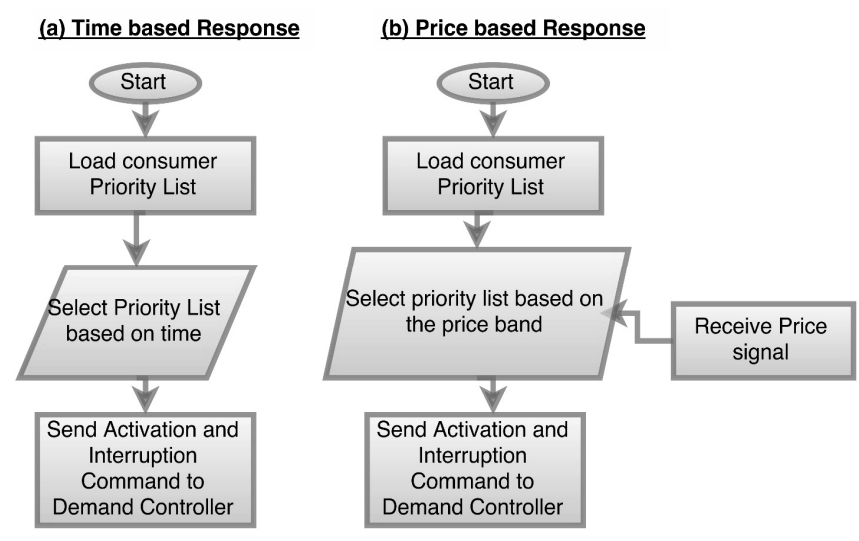

Fig. 1. Time based and Price based Demand Response flow diagram in literature [4] which constitute the three and half decades of demand side management evolution.

\section{B. Fuzzy logic based DR}

The fuzzy logic was first proposed by Lotfi Zadeh [14]. Fuzzy systems are considered as universal approximates that quantify non-precise inputs to obtain solutions that are based on rules. Essentially, fuzzy logic facilitates (fuzzy) controllers to take into account of complex inputs such as human comfort, which maybe based purely on human reasoning. These inputs are difficult to formulate analytically. The Fuzzy controller modelled in this paper is provided in fig 2 . Inputs are consumer profiling, smart meter data and environment condition data. Inputs and outputs are formulated using conventional membership functions. The Mamdani method [14] of fuzzy inference is utilized to map input-output relationships that are based on a user defined set of rules. The output of this fuzzy system is also a fuzzy set of allowed load or scheduled load. The crisp value for specific inputs is obtained by defuzzifying the fuzzy set by a centroid defuzzification technique and provides a numerical value in

$$
x^{*}=\frac{\int \mu_{i}(x) x d x}{\int \mu_{i}(x) d x}
$$

Where $x^{*}$ is the defuzzified (numerical) output, $\mu_{i}(x)$ is the $i^{\text {th }}$ output membership function and $x$ is the output variable. The fuzzy rules and membership function values are formulated based on the previous experience and knowledge from the grid. Fuzzy systems can be modelled to represent any type of relations, especially human behaviour dependent relations. As an example case, consider customer comfort and its dependency on the ambient temperature. Fuzzy modelling can facilitate the interest of a customer to participate in DR while considering special situations such as a European cup football final etc. In this regard an accurate DR may be achieved without affecting customer comfort.

\section{MODEL DESCRIPTION}

\section{A. Direct Load Method}

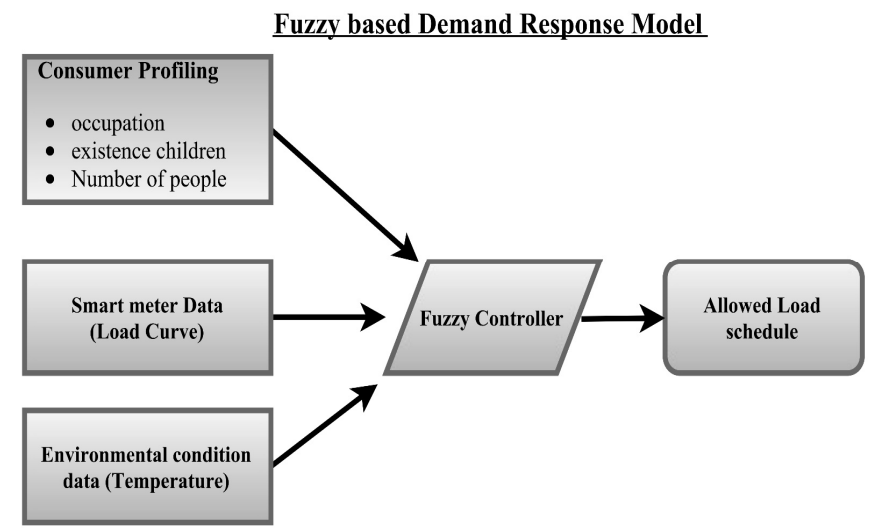

Fig. 2. Fuzzy logic based Demand Response Model

To implement direct load based DR through either the price based and time based mothods, a list of domestic appliances is required. As an arbitrary representation, the appliances in 6 households are considered and their respective power ratings 
were obtained from a general survey as provided in table 1 . The list can be extended and considered according to the need and type of appliances installed in any installation. We also assume that these appliances are smart and can communicate with a master controller without much time lag being incurred. In a price based DR, luminaires represent the non-dispatchable load and all other loads are considered dispatchable or controllable. In time based DR, specific loads are considered nondispatchable because that load is considered unavoidable at that specific time. The customer can set the required load priority at their own discretion. The priority list is given in table II for price based DR. Status 1 signifies 'on' position and 0 signifies 'off'. When a price signal is received this priority list is interrogated to establish the possible schedule with operating signals sent to the DR controller accordingly.

Table 3 provides a time based priority list, which is subsequently employed to develop the time based DR schedule. The schedule is organised for 3-hour intervals. The ' $x$ ' in Table 3 represents non-dispatchable load and dispatchable loads are scheduled according to the priority. The value'0' implies the load is switched off at the particular interval of time. The allowed load in each interval is obtained by selecting the respective priority level during each interval of time. For example, during the $3^{\text {rd }}$ interval, dispatchable loads 1 and 2 are allowed to operate and 3 and 4 are switched off. Similarly, each interval has a specific schedule according to the DR controller.

TABLE I

LOAD AND RESPECTIVE POWER CONSUMPTION

\begin{tabular}{cc}
\hline Loads & Rated Power(W) \\
\hline Light & 300 \\
\hline TV & 200 \\
\hline Washing Machine & 500 \\
\hline Water Pump & 1000 \\
\hline Heating & 1000 \\
\hline Computer & 150
\end{tabular}

TABLE II

PRICE BASED PRIORITY LIST

\begin{tabular}{ccccccc}
\hline \multirow{2}{*}{ Equipment } & \multicolumn{7}{c}{ Price Band } \\
\cline { 2 - 8 } & I & II & III & IV & V & VI \\
\hline Light & 1 & 1 & 1 & 1 & 1 & 1 \\
\hline TV & 1 & 1 & 1 & 1 & 0 & 0 \\
\hline Washing Machine & 1 & 1 & 0 & 0 & 0 & 0 \\
\hline Water Pump & 1 & 1 & 0 & 0 & 0 & 0 \\
\hline Heating & 1 & 1 & 1 & 1 & 1 & 0 \\
\hline Computer & 1 & 1 & 1 & 1 & 1 & 0 \\
\hline
\end{tabular}

\section{B. Fuzzy Logic Based DR}

The advantage of a fuzzy control algorithm over other algorithms is the ablity to model non deterministic inputs using non numeric linguistic values. Human characteristics, behaviour and response are easily represented by linguistic values rather than using crisp Boolean logic. Furthermore, fuzzy logic is able to represent intermediate values similar to human decision making.

Fuzzy inference systems (FIS) help to model human knowledge into linguistic 'if-then' conditional statements; without using precise quantitative analysis. These 'if-then' statements are called upon as rules in the fuzzy system. Inputs
TABLE III

TIME BASED PRIORITY LIST

\begin{tabular}{ccccccccc}
\hline \multirow{2}{*}{ Equipment } & \multicolumn{8}{c}{ Time (Interval of 3 Hours) } \\
\cline { 2 - 9 } & $\mathbf{1}$ & $\mathbf{2}$ & $\mathbf{3}$ & $\mathbf{4}$ & $\mathbf{5}$ & $\mathbf{6}$ & $\mathbf{7}$ & $\mathbf{8}$ \\
\hline Light & 0 & 0 & $\mathrm{x}$ & 0 & 0 & 1 & $\mathrm{x}$ & 3 \\
\hline TV & 0 & 0 & 1 & 0 & 0 & 4 & $\mathrm{x}$ & 4 \\
\hline $\begin{array}{c}\text { Washing } \\
\text { Machine }\end{array}$ & 2 & 2 & 4 & 2 & 4 & 6 & 3 & 6 \\
\hline Water Pump & 1 & 1 & 3 & 1 & 3 & 5 & 2 & 5 \\
\hline Heating & $\mathrm{x}$ & $\mathrm{x}$ & $\mathrm{x}$ & 0 & 1 & 2 & $\mathrm{x}$ & 1 \\
\hline Computer & 0 & 0 & 2 & 0 & 2 & 3 & 1 & 2 \\
\hline
\end{tabular}

and outputs, which are modelled as linguistic variables, are characterized by their appropriate membership function with attributes defined as linguistic values. Typical membership functions are representations of the input characteristics and vary smoothly without sharp boundaries between 0.1 and 1 . Inputs are combined using logical and, or, not operations. In general, first input values are fuzzfied or mapped to their respective membership functions and the degree of membership is assigned. Then statements of rule assign these partial memberships to the associated output membership functions after the logical operation of inputs. The outputs are weighted according to the strength of each rule. The final output is calculated using respective defuzzification techniques. Here the centroid method is utilized. The inputs of the fuzzy DR controller are time of day, the load forecast or load demand, temperature deviation, and temporal availability. Outputs are the signal(s) of allowed load and the 'on' or 'off' signal for the air conditioning system. The input and output are modelled using only two types of membership function; triangular and trapezoidal membership functions. The demand response model implemented through the fuzzy toolbox in MATLAB is provided in fig 3. The output of the controller is defined using 465 rules which are defined based on the experience and intuition of the modeller. Rules are basically linguistic 'if- then' statements. The number of rules possible in a fuzzy controller is defined by the possible number of combinations of inputs and outputs. Even though more than 1000 rules could have been formulated, only feasible rules have been considered here. Some examples of these rules are provided in the appendix (section VI) for reference. Temporal availability is modelled as a separate fuzzy controller whose output indicates the presence of people in the house. The deciding parameter or the inputs considered are type of occupation, number of occupants in the house, the existence of children in the house and the time of day. The output of the temporal fuzzy controller is provided as the input to the fuzzy DR controller. The temporal fuzzy controller is defined using 98 rules. Again, some examples of these rules are listed in the

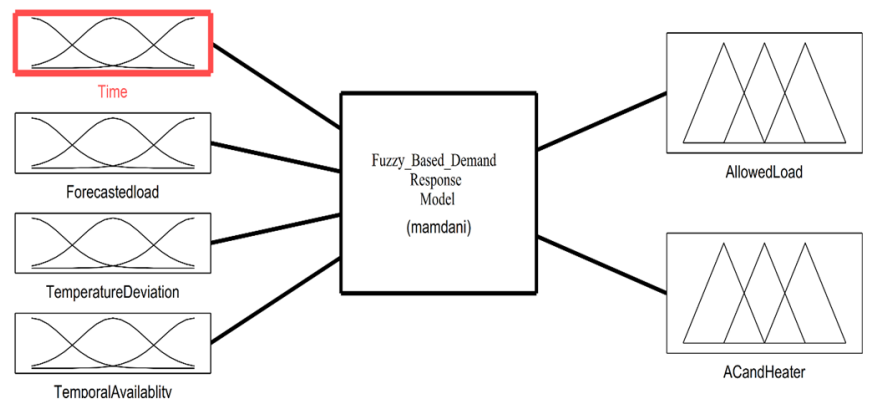

Fig. 3. Fuzzy DR controller model implemented in MATLAB 
appendix. The temporal fuzzy block diagram is provided in fig 4.

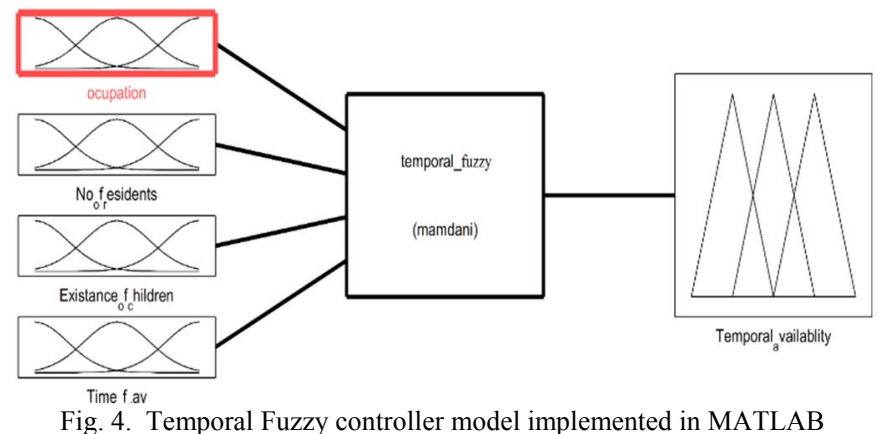

\section{RESULTS}

To demonstrate the simulation model, an example case study is considered and the results are contextualised accordingly. As the model is not yet validated practically, the process is to consider a large number of inputs to the model so that comprehension, analysis, and interpretation of the results are readily achievable.

To implement the price based DLC method, a price signal is considered which is assumed to be available prior to scheduling. In this particular study, the price signal is formulated according to fig 5. According to the price at a given time, the price band is selected. Based on the price band, the output is obtained. The price band, as shown in table II, is divided into 6 bands in increasing order from 1 to 6 . Where 6 is the highest price and 1 being the lowest.

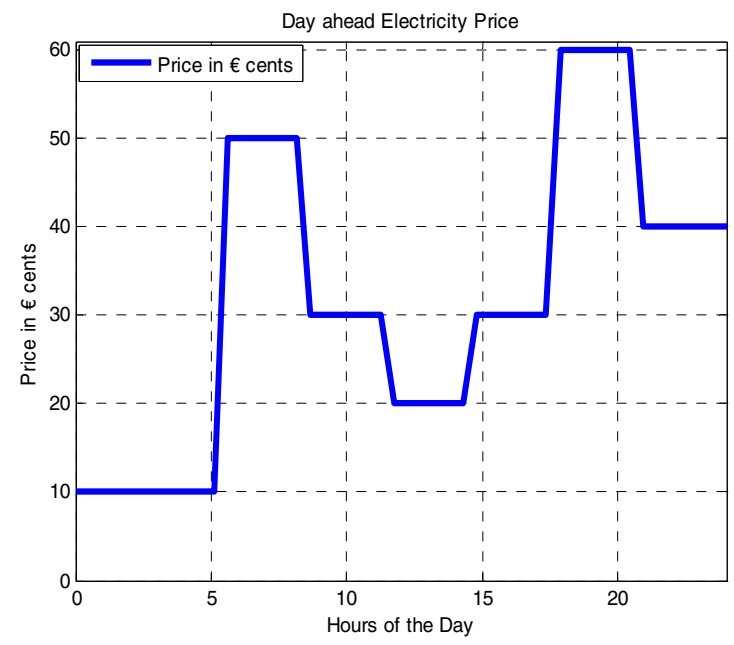

Fig. 5. Price signal for a day

The price in each band is decided according to the market price trend decided by the market/utility. The demand response for a day corresponding to the price signal is provided in fig 6 . The load demand is provided by the load demand possibility of a house decided by data collected from a simple survey. Fig 6 shows the load demand curve for a particular day. When the price is high during the 6-9am period (as provided in fig 5), the load is reduced from $3.1 \mathrm{~kW}$ demand to lower than $1.5 \mathrm{~kW}$. This type of DR is not concerned with the customer comfort and only deals with reduction of the load during high price. The method is purely based on cost savings and will be profitable for both customers and the utility at the same time.

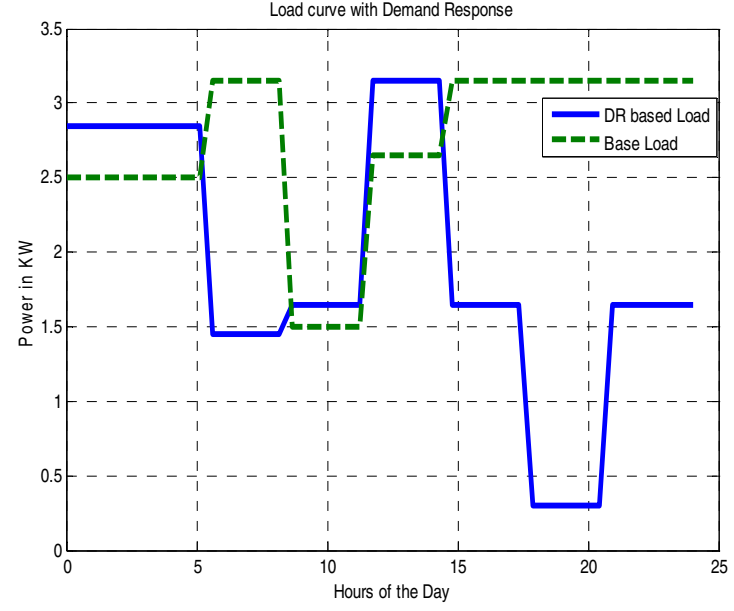

Fig. 6. Electricity price for Price based DR

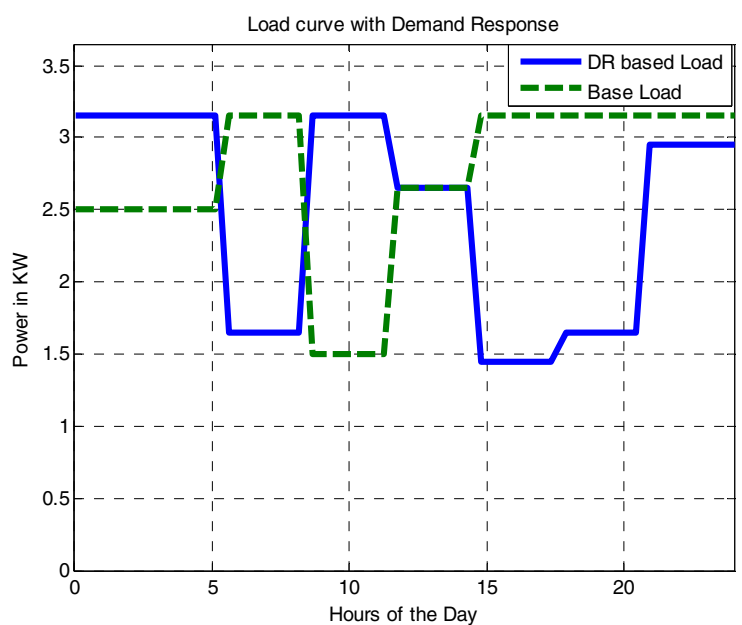

Fig. 7. Base load and demand response (Time based)

Time based demand response is obtained by scheduling the load based on a respectively assigned time based priority list. The method primarily depends on the general nature of peak curve. The peak is assumed to be in the morning and evening hours. During these times a specific set of equipment (appliances) are allowed to work which are considered to be inevitably required at those particular times. During the evening peak which is supposed to be during 6-9 PM the load is reduced close to $1.5 \mathrm{~kW}$ from $3.1 \mathrm{~kW}$. This method doesn't consider peak magnitude and works like a strict controller bounded by a specific time based rules without any alterations. The method is useful if the load reduction required is small and the peak time is constant. In all another cases however, the method is not so useful. Moreover, this method, like price based DR, is not considerate of customer comfort.

To implement a fuzzy based controller, the temporal availability needs to be modelled at the onset. For the study here, a basic household data selected is given in table IV. The temporal availability also depends on the time of the day since, during a normal night, temporal availability is supposed to be full and during a 'normal' day, it depends on various factors like occupation, the existence of children etc. The output of temporal availability is used for scheduling load using demand response. 
TABLE IV

STATIC INPUT DATA OF HOUSEHOLD

\begin{tabular}{ll}
\hline \multicolumn{2}{l}{ Basic data of individual household } \\
\hline Number of people in house & 3 \\
\hline Existence of children & Yes \\
\hline Occupation & Full time \\
\hline
\end{tabular}

The input, forecasted load is obtained from the utility. In this analysis, power demand of a household for typical day is used for scheduling. The demand of a 3 bedroom house is used which has a peak demand close to $5 \mathrm{~kW}$ is considered as a base case for the fuzzy based DR analysis. The load curve shows that the demand is high during the morning 6am to 9am and evening $6 \mathrm{pm}$ to $10 \mathrm{pm}$. The demand response is designed to regulate the load during this period to obtain a lower peak. Since customer comfort is considered, the switching 'on' and 'off' of the air-conditioning load is only dependent on temperature deviation and temporal availability. The temperature data for the location of house located in Dublin is obtained from the meteorological data centre. The temperature for a normal day, warm day and cold day is obtained for finding the variation of DR depending on customer comfort for the same load demand. These temperature data are plotted together in fig 8. The demand response for these temperatures for the occupants and load demand is shown in fig 9. It can be observed that with ambient temperature change the DR based load also changes for same temporal availability and load demand. This decision override is achieved by the fuzzy controller which is working on the linguistic rules which state that the air-conditioner should be 'on' if the temperature is high or the associated heater should be 'on' if temperature is low and irrespective of the load peak if the temporal availability is 'yes'. The justification for this is that the use of temperature regulators purely depend on consumer comfort. Even then, the load during the peak demand is reduced and demand response based load management is achieved. The method therefore places an increased burden on the utility by not facilitating complete load reduction during the peak period. That said however, the reduction is still formidable, and when it is implemented by large number of customer it will make an impressive difference in load demand.

Fig 10 shows the variation of DR with the change in temporal availability. When the presence of the customer changes there is a specific change in load consumption as expected in the normal case.

Further, this can be quantified by the fact that the temperature regulators are not required when the customer is not available at the house. This result is obtained for a warm day so that it can be compared with the result in fig 9 for complete comprehension of DR depending on temporal availability. The results suggest therefore, that DR programs can effectively reduce load/demand during peak demand periods and as illustrated in figs. 9 and 10. DR offers further benefits. These benefits will manifest as cost reduction, supply reliability increase, market efficiency, customer service improvement, environment sustainability improvement and market power mitigation [15].

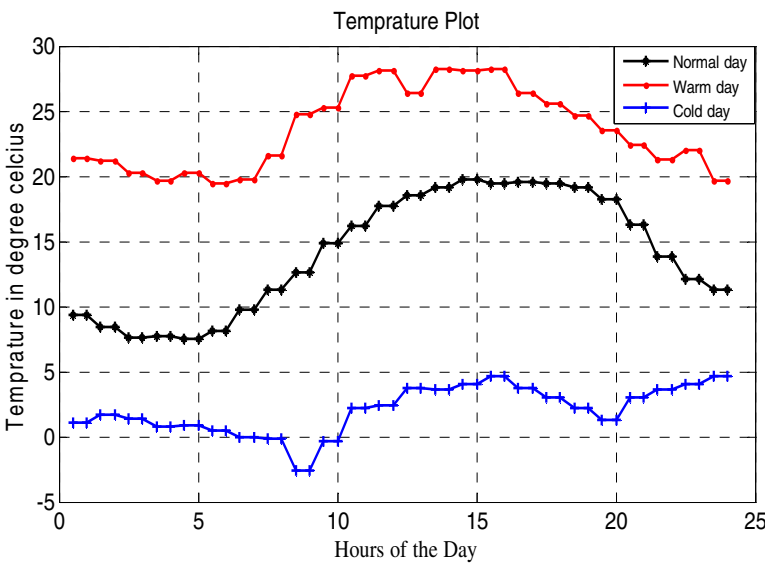

Fig. 8. Temperature curve for warm, cold and normal day (Data from http://www.met.ie)

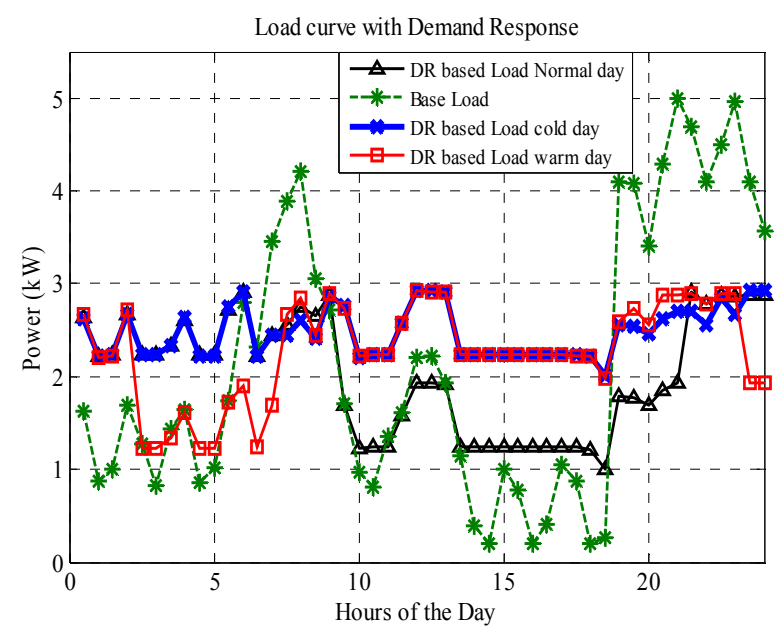

Fig. 9. DR for different temperature

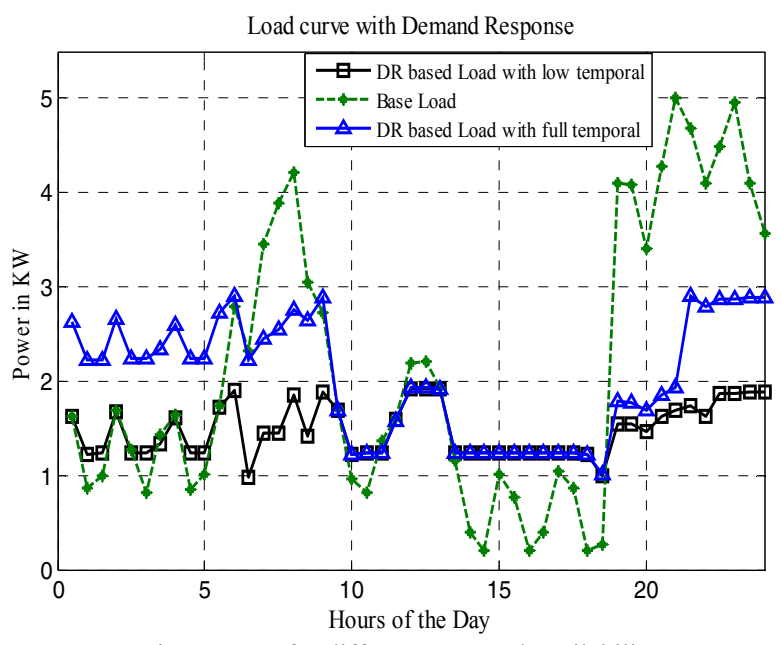

Fig. 10. DR for different temporal availability

\section{CONCLUSION}

The paper describes the modelling of simple DLC-DR and DR using a fuzzy system approach which is typically a rational decision making model.

The DLC based DR model presented here is able to reduce the consumption but at the cost of customer comfort. By utilizing the non-deterministic characteristics of fuzzy control algorithms, a DR controller is implemented to account for 
customer comfort and hence schedules the temperature control equipment independent of DR. The model also depicts the impact of DR with change in temperature and temporal availability.

The modelling considered can be used to implement DR in the context of substation control to study the effect of DR in a macro scale. Further, including the renewable energy production could enable load scheduling according to the availability of renewable energy, which will reduce the energy consumption from conventional sources.

\section{REFERENCES}

[1] M.H. Albadi, E.F. El-Saadany, A summary of demand response in electricity markets, Electric Power Systems Research, Volume 78, Issue 11, November 2008, Pages 1989-1996, ISSN 0378-7796,

[2] G. Hug, S. Kar and C. Wu, "Consensus + Innovations Approach for Distributed Multiagent Coordination in a Microgrid," in IEEE Transactions on Smart Grid, vol. 6, no. 4, pp. 1893-1903, July 2015.

[3] M. Mollahassani-pour, A. Abdollahi and M. Rashidinejad, "Investigation of Market-Based Demand Response Impacts on Security-Constrained Preventive Maintenance Scheduling," in IEEE Systems Journal, vol. 9, no. 4 , pp. $1496-1506$, Dec. 2015

[4] Goran Strbac, Demand side management: Benefits and challenges, Energy Policy, Volume 36, Issue 12, December 2008, Pages 4419-4426

[5] Assessment of demand response and advanced metering, FERC, Washington, DC, USA, Staff Report, Feb. 2011. [Online]. Available: http://www.FERC.gov3.

[6] C. Chen, J. Wang and S. Kishore, "A Distributed Direct Load Control Approach for Large-Scale Residential Demand Response," in IEEE Transactions on Power Systems, vol. 29, no. 5, pp. 2219-2228, Sept. 2014.

[7] S. Lu et al., "Centralized and decentralized control for demand response," Innovative Smart Grid Technologies (ISGT), 2011 IEEE PES, Hilton Anaheim, CA, 2011, pp. 1-8.

[8] J. A. Short, D. G. Infield and L. L. Freris, "Stabilization of Grid Frequency Through Dynamic Demand Control," in IEEE Transactions on Power Systems, vol. 22, no. 3, pp. 1284-1293, Aug. 2007.

[9] A. Naeem, A. Shabbir, N. Ul Hassan, C. Yuen, A. Ahmad and W. Tushar, "Understanding Customer Behavior in Multi-Tier Demand Response Management Program," in IEEE Access, vol. 3, no. , pp. 2613-2625, 2015

[10] H. Salehfar, P. J. Noll, B. J. LaMeres, M. H. Nehrir and V. Gerez, "Fuzzy logic-based direct load control of residential electric water heaters and air conditioners recognizing customer preferences in a deregulated environment," Power Engineering Society Summer Meeting, 1999. IEEE, Edmonton, Alta., 1999, pp. 1055-1060 vol.2

[11] Babu, P. Ravi, and Rashmi Reddy. "DSM techniques and Fuzzy logic application to Air conditioner-a case study." 8th WSEAS International Conference on POWER SYSTEMS (PS 2008), Santander, Cantabria, Spain. 2008.

[12] Z. Wang, R. Yang and L. Wang, "Multi-agent control system with intelligent optimization for smart and energy-efficient buildings,"
IECON 2010 - 36th Annual Conference on IEEE Industrial Electronics Society, Glendale, AZ, 2010, pp. 1144-1149.

[13] O. A. Sianaki, O. Hussain, T. Dillon and A. R. Tabesh, "Intelligent decision support system for including consumers' preferences in residential energy consumption in smart grid", 2010 IEEE Second International Conference on Computational Intelligence, Modelling and Simulation, pp. 154-159

[14] T. J. Ross, Fuzzy logic with engineering applications. United States: John Wiley \& Sons, 2010.

[15] Peak Load Management Alliance, "Demand Response: Principles for Regulatory Guidance,” February 2002.

\section{APPENDIX}

Fuzzy rules for temporal calculation

1. If (occupation is no and No of residents is one and Existence of children is yes and Time of day is $\mathbf{0 - 3 h r s ) ~ t h e n ~ ( T e m p o r a l ~ a v a i l a b i l i t y ~}$ is yes)

2. If (occupation is no and No of residents is more than $\mathbf{2}$ and Existence of children is yes and Time of day is $\mathbf{0 - 3 h r s ) ~ t h e n ~ ( T e m p o r a l ~}$ availability is yes)

3. If (occupation is no and No of residents is more than $\mathbf{2}$ and Existence of children is yes and Time of day is one) then (Temporal availability is yes)

4. If (occupation is part time and No of residents is one and Existence of children is no and Time of day is 6-9hrs) then (Temporal availability is yes)

5. If (occupation is part time and No of residents is more than $\mathbf{2}$ and Existence of children is no) and Time of day is $\mathbf{0 - 3 h r s}$ ) then (Temporal availability is yes)

6. If (occupation is full time and No of residents is one and and Existence of children is no Time of day is $\mathbf{1 5 - 1 8 h r s ) ~ t h e n ~ ( T e m p o r a l ~}$ availability is no)

\section{Fuzzy rules for Demand response}

1. If Time is off peak and Forecasted load is Low Medium and Temperature Deviation is cold) and Temporal Availability is yes) then (Allowed Load is 3)(AC and Heater is Heater)

2. If (Time is off peak and Forecasted load is Low Medium and Temperature Deviation is moderate and Temporal Availability is yes) then Allowed Load is 4)(AC and Heater is no action)

3. If (Time is off peak and Forecasted load is Medium and Temperature Deviation is cold and Temporal Availability is no) then Allowed Load is 6)(AC and Heater is no action)

4. If (Time is off peak and Forecasted load is Medium and Temperature Deviation is hot and Temporal Availability is yes) then Allowed Load is 7)(AC and Heater is AC)

5. If (Time is off peak and Forecasted load is high and Temperature Deviation is cold and Temporal Availability is yes) then Allowed Load is 7)(AC and Heater is Heater)

6. If (Time is evening peak and Forecasted load is Medium and Temperature Deviation is cold and Temporal Availability is no) then Allowed Load is 2)(AC and Heater is no action) 